\title{
Follow-up at 1 year and beyond of women with gestational diabetes treated with insulin and/or oral glucose-lowering agents: a core outcome set using a Delphi survey
}

\author{
Delia Bogdanet ${ }^{1}$ (D) Catriona Reddin ${ }^{1} \cdot$ Esther Macken $^{1} \cdot$ Tomas P. Griffin $^{1} \cdot$ Narjes Fhelelboom $^{1} \cdot$ Linda Biesty $^{1}$. \\ Shakila Thangaratinam ${ }^{2}$. Eugene Dempsey ${ }^{3} \cdot$ Caroline Crowther $^{4} \cdot$ Sander Galjaard $^{5} \cdot$ Michael Maresh $^{6}$. \\ Mary R. Loeken ${ }^{7,8}$ • Angela Napoli ${ }^{9}$ Eleni Anastasiou ${ }^{10}$ • Eoin Noctor ${ }^{11}$ - Harold W. de Valk ${ }^{12}$. \\ Mireille N. M. van Poppel ${ }^{13}$. Andrea Agostini ${ }^{14}$. Cheril Clarson ${ }^{15,16}$. Aoife M. Egan ${ }^{17}$ - Paula M. O'Shea ${ }^{1}$. \\ Declan Devane $^{1}$. Fidelma P. Dunne ${ }^{1}$
}

Received: 28 January 2019 / Accepted: 21 May 2019 / Published online: 4 July 2019

(C) The Author(s) 2019

\begin{abstract}
Aims/hypothesis Gestational diabetes mellitus (GDM) is linked with a higher lifetime risk for the development of impaired fasting glucose, impaired glucose tolerance, type 2 diabetes, the metabolic syndrome, cardiovascular disease, postpartum depression and tumours. Despite this, there is no consistency in the long-term follow-up of women with a previous diagnosis of GDM. Further, the outcomes selected and reported in the research involving this population are heterogeneous and lack standardisation. This amplifies the risk of reporting bias and diminishes the likelihood of significant comparisons between studies. The aim of this study is to develop a core outcome set (COS) for RCTs and other studies evaluating the long-term follow-up at 1 year and beyond of women with previous GDM treated with insulin and/oral glucose-lowering agents.

Methods The study consisted of three work packages: (1) a systematic review of the outcomes reported in previous RCTs of the follow-up at 1 year and beyond of women with GDM treated with insulin and/or oral glucose-lowering agents; (2) a three-round online Delphi survey with key stakeholders to prioritise these outcomes; and (3) a consensus meeting where the final COS was decided.

Results Of 3344 abstracts identified and evaluated, 62 papers were retrieved and 25/62 papers were included in this review. A total of 121 outcomes were identified and included in the Delphi survey. Delphi round 1 was emailed to 835 participants and 288 $(34.5 \%)$ responded. In round 2, 190 of $288(65.9 \%)$ participants responded and in round 3, 165 of $190(86.8 \%)$ participants responded. In total, nine outcomes were selected and agreed for inclusion in the final COS: assessment of glycaemic status; diagnosis of type 2 diabetes since the index pregnancy; number of pregnancies since the index pregnancy; number of pregnancies with a diagnosis of GDM since the index pregnancy; diagnosis of prediabetes since the index pregnancy; BMI; post-pregnancy weight retention; resting blood pressure; and breastfeeding.

Conclusions/interpretation This study identified a COS that will help bring consistency and uniformity to outcome selection and reporting in clinical trials and other studies involving the follow-up at 1 year and beyond of women diagnosed with GDM treated with insulin and/or oral glucose-lowering agents during pregnancy.
\end{abstract}

Keywords Core outcome set · Gestational diabetes mellitus · Insulin · Oral hypoglycaemic agents

Electronic supplementary material The online version of this article (https://doi.org/10.1007/s00125-019-4935-9) contains peer-reviewed but unedited supplementary material, which is available to authorised users.

Delia Bogdanet

deliabogdanet@gmail.com

Sander Galjaard

s.galjaard@erasmusmc.nl

Extended author information available on the last page of the article
Abbreviations

COMET Core Outcome Measures in

Effectiveness Trials Initiative

COS Core outcome set

COS-STAR Core Outcome Set STAndards for

Reporting

GDM Gestational diabetes mellitus 


\section{Research in context}

\section{What is already known about this subject?}

- Gestational diabetes is associated with a significant lifetime risk of developing type 2 diabetes, the metabolic syndrome, cardiovascular disorders, postpartum depression and tumours

- A core outcome set (COS) is the minimum set of outcomes that should be measured and reported in all clinical trials and other studies

\section{What is the key question?}

- What is the COS that should be measured and reported for trials and other studies evaluating the long-term follow-up at 1 year and beyond of women with previous GDM treated with insulin and/or oral glucose-lowering agents?

What are the new findings?

- Following a systematic review of the literature, a three-round online Delphi survey and a consensus meeting, nine outcomes were included in the $\operatorname{COS}$

\section{How might this impact on clinical practice in the foreseeable future?}

- $\mathrm{A} C O S$ in the follow-up of women with gestational diabetes has the potential to reduce reporting bias and increase consistency in outcome reporting, thus facilitating evidence-based synthesis and, ultimately, increase the quality of research and healthcare delivery to women with previous gestational diabetes

\section{IADPSG International Association of Diabetes in Pregnancy Study Group SAG Study advisory group}

\section{Introduction}

The prevalence of gestational diabetes mellitus (GDM) is increasing worldwide and ranges between $5.2 \%$ and $40.4 \%$ across countries; this wide variability reflects multiple factors such as BMI, ethnicity, country income, and also the diagnostic criteria used [1]. Based on the International Association of Diabetes in Pregnancy Study Group (IADPSG) criteria, the European prevalence of GDM is approximatively $13 \%$ [2, 3]. Medical nutritional therapy (diet and exercise) is the first step in the treatment of GDM [4]. However, if the desired glycaemic goals are not achieved, pharmacological treatment will be required.

GDM is linked with a substantial lifetime risk of developing type 2 diabetes. A meta-analysis conducted on studies published over the last 50 years showed that women with a history of GDM have a higher risk of developing type 2 diabetes (RR 7.4) compared with women with normal glucose tolerance (NGT) in pregnancy [5]. Women with previous GDM are more likely to develop the metabolic syndrome [6] and cardiovascular disorders [7] in later life. Several studies have shown that women with GDM have a higher risk of developing postpartum depression $[8,9]$. There is also a growing body of literature associating a history of GDM with the development of tumours, particularly breast and endometrial tumours [10].

Women with GDM should be screened for persistent diabetes or impaired fasting glucose and/or impaired glucose tolerance at 6-12 weeks postpartum using non-pregnancy criteria [11]. However, there is no standardised approach to the long-term follow-up of women with a previous GDM diagnosis. The results of clinical trials evaluating comparable interventions are usually summarised in systematic reviews and meta-analyses that provide the basis for guidelines and treatment recommendations. However, there is little consistency in outcome selection and reporting in clinical trials involving this population. This inconsistency raises concern for possible outcome selection bias, makes significant research synthesis difficult and limits the ability to combine the findings of individual studies into summary estimates. One way to overcome this is to develop a core outcome set (COS).

A COS is the minimum set of outcomes that should be consistently measured and reported in all clinical trials (and other studies). However, this does not restrict researchers from adding additional outcomes. A minimum set of outcomes will provide greater uniformity of reporting in clinical trials and more data to impact meta-analyses. Also, a COS will reduce study heterogeneity and the risk of reporting bias by consistently measuring and reporting these outcomes.

The Core Outcome Measures in Effectiveness Trials (COMET) initiative aims to standardise outcome reporting 
in trials, facilitates participation of diverse experts undertaking research and minimises duplication of work $[12,13]$. The Core Outcome Set STAndards for Reporting (COS-STAR) is a checklist designed to be applicable regardless of the consensus methods used to develop the COS and the various participant groups [14]. The COS-STAR checklist provides guidance for minimal COS study reporting and its purpose is to promote the transparency and completeness of reporting in all COS studies. The CoRe Outcomes in Women's and Newborn health $(\mathrm{CROWN})$ initiative encourages the development of COSs in women's and newborns' health.

The aim of this study was to develop a COS for trials and other studies evaluating the long-term follow-up at 1 year and beyond of women with previous GDM treated with insulin and/oral glucose-lowering agents. This study focuses only on women with GDM treated with insulin and oral glucoselowering agents as this population has more severe glucose abnormalities and are more likely to progress to type 2 diabetes, obesity and the metabolic syndrome $[15,16]$.

\section{Methods}

This study is registered in the COMET database [17]. Ethical approval for the study was obtained from the Galway University Hospitals Research Ethics Committee (reference CA 1905).

The three work packages of the study were: (1) a systematic review of literature that identified all the outcomes reported in clinical trials that involved the long-term follow-up of this population; (2) a Delphi survey in which all outcomes were scored and prioritised by key stakeholder groups to provide a preliminary list of final outcomes; and (3) a consensus meeting where the final list of outcomes was decided.

Systematic review Using a broad-based search strategy, the following databases were searched for relevant studies between October 2017 and February 2018: Cochrane Central Register of Controlled Trials (CENTRAL), the Cumulative Index to Nursing and Allied Health Literature (CINAHL), PubMed, EMBASE and Web of Science. ClinicalTrials.gov was also searched for relevant ongoing trials. The reference lists of all included studies were searched for additional studies not retrieved from the electronic database search. There was no time restriction on the date of publication of the studies. There was no language restriction applied to the search strategy. Only RCTs and RCT follow-up studies were included in the systematic review (an example of the search strategy is presented in the electronic supplementary material [ESM] Methods).

In step 1, all identified study titles were reviewed and ineligible studies excluded (F.P. Dunne and D. Bogdanet). In step 2, the remaining studies were appraised by two reviewers (F.P. Dunne and D. Bogdanet) who independently assessed the titles and abstracts of each study included at this stage. Full texts of studies meeting the inclusion criteria and studies for which there was uncertainty regarding inclusion at the title/ abstract screening stage were retrieved and reviewed independently (F.P. Dunne and D. Bogdanet). The same two authors extracted the data independently, reviewed the data together, assessed consensus and ensured that all outcomes were identified. Following review by F.P. Dunne, D. Devane, D. Bogdanet, L. Biesty, A. M. Egan and P. M. O'Shea (the study advisory group [SAG]), extracted outcomes were grouped under the following domains: laboratory tests, clinical conditions, physiological variables, diet and exercise, psychological variables and other.

Delphi method We conducted a three-round eDelphi survey [18]. This facilitated international participation. Questionnaires were completed online using SurveyMethods software (www. surveymethods.com, SurveyMethods, Dallas, TX, USA, accessed 9 May 2018). Full details of our methods are given in Bogdanet et al (2019) [19] and are described briefly below.

The stakeholder groups comprised: women with a previous diagnosis of GDM, endocrinologists, diabetes nurses, obstetricians, midwives, paediatricians, neonatologists, general practitioners, practice nurses, dietitians, physiotherapists, researchers with expertise in gestational diabetes, policy makers and others (which included clinicians with expertise in gestational diabetes from specialties other than endocrinology and obstetrics, epidemiologists, clinical biochemists and healthcare assistants).

Invitation emails were sent to societies and individual members of the IADPSG, Diabetes Ireland, Irish Endocrine Society (IES), IDF, International Federation of Gynecology and Obstetrics (FIGO), European Board and College of Obstetrics and Gynaecology (EBCOG), Irish Nutrition and Dietetic Institute (INDI), Association of Clinical Biochemists in Ireland (ACBI), Irish Institute of Obstetricians and Gynaecologists, Saolta Healthcare Group (Ireland), EASD, Diabetic Pregnancy Study Group (DPSG) of the EASD and the Royal College of Physicians Ireland (RCPI) (divisions of Endocrinology, Obstetrics and Endocrinology and Paediatrics). All participants were asked to forward the invitation to others whom they regarded as having the required expertise. Additionally, women with a history of GDM were contacted through their clinic by the authors and by additional study participants and, following consent, were forwarded the survey link or given a printed form of the survey. Women with GDM were from a number of clinics; the final group who participated in the consensus meeting were from the Galway clinic.

Study participants gave informed consent prior to the submission of any answers and the following information was also requested: name, email address, sex, stakeholder group and country of residence. Participants were given information about the study and about COSs. Participants were encouraged to complete the eDelphi questionnaire in each round. 
An email reminder was sent to anyone who did not respond after 7 and 14 days and also 3 days and 1 day before the end of the round.

In the first round of the survey, all the outcomes identified in the systematic review were presented to the participants grouped by domain. The study participants were asked to rate each outcome on a nine-point Likert scale (1-3 limited importance; 4-6 important but not critical, 7-9 critical). We provided all participants with plain English explanations of the outcomes included in the survey. Participants were invited to suggest additional relevant outcomes (no limit to the number of outcomes suggested) using free-text responses. If two or more study participants nominated an outcome, that outcome was included in round 2 of the survey.

All stakeholder groups were grouped into three broader groups, i.e. clinicians, women with a previous diagnosis of GDM and researchers/policy makers. Descriptive statistics were used to summarise the results from round 1 . We sent individual results, the results of each stakeholder group and the results of the total group to each study participant. All outcomes including the additional outcomes suggested in round 1 (by two or more participants) were carried forward to round 2 . All respondents to round 1 were invited to participate in round 2 and asked to re-rate the outcomes. All outcomes that scored 7-9 on the Likert scale in $\geq 70 \%$ of answers and $1-3$ in $<15 \%$ of answers were carried forward to round 3 . Each participant who completed round 2 was emailed their individual results and the results of each stakeholder group and the total group and was invited to participate in round 3 and re-score retained outcomes. Outcomes were classified as 'consensus in' ( $\geq 70 \%$ participants scoring as $7-9$ and $<15 \%$ scoring as $1-3$ ) or 'consensus out' ( $\geq 70 \%$ scoring as $1-3$ and $<15 \%$ scoring as $7-9$ ). The 'consensus in' and borderline outcomes were brought forward to the consensus meeting.

Consensus meeting The consensus meeting involved representatives from each stakeholder group. The participants discussed each outcome brought forward from round 3. If necessary, the outcomes were grouped or renamed in order to facilitate dissemination and usefulness. At the end of the discussion, each participant voted 'outcome in' or 'outcome out' using the app Poll Everywhere (San Francisco, CA, USA, accessed 27 September 2018) on their electronic device, thus concealing their identity. Outcomes that scored over $70 \%$ 'outcome in', were included in the final COS.

\section{Results}

A total of 3344 titles and abstracts were identified. Following review of the title and/or abstracts, 62 full text papers were retrieved and assessed for eligibility. A further 37 papers were excluded following full text assessment, leaving 25 papers in the review (ESM Table 1). Following data extraction, 121 individual outcomes were identified. Following the SAG meeting, similar outcomes were combined, leaving a final 116 outcomes to be grouped and included in round 1 (ESM Fig. 1).

The first round of the Delphi survey was sent to 835 participants (societies and individual members). At the end of round 1, there were 288 respondents $(34.5 \%)$ representing 33 countries and five continents (Table 1). A total of $73 \%$ of the respondents were female. The distribution of answers throughout the stakeholder groups in each of the three rounds is presented in Table 1. An additional ten outcomes were suggested by two or more study participants and were included in round 2 (ESM Table 2).

Round 2 participants were asked to rate 126 outcomes grouped as described in the Methods section. Round 2 was completed by $65.9 \%$ of the round 1 responders (190 participants). Similar to round 1, there was a female predominance among responders (73.7\%). The distribution of answers amid stakeholder groups was similar to round 1 (Table 1) (clinicians $82.6 \%$, women with a history of GDM $10 \%$, researchers/ policy makers $7.4 \%$ ). All outcomes that scored $7-9$ on Likert scale in $\geq 70 \%$ and $1-3$ in $<15 \%$ by study participants were brought forward to round $3(n=34)$. The percentage of participants who voted $1-3,4-6$ or $7-9$ on each outcome at the end of round 2 is presented in Table 2.

Round 3 was completed by 165 participants $(86.8 \%)$. Similar to round 2, outcomes were brought forward when $70 \%$ or more participants scored the outcome as $7-9$ and $<15 \%$ participants scoring as $1-3$. In total, 30 outcomes went through the consensus meeting (ESM Table 3).

Consensus meeting The consensus meeting involved 20 participants, a chairperson and an administrator. The stakeholder groups included four women with a history of gestational diabetes, one diabetes nurse specialist, two midwives, one policy maker, two paediatricians, one clinical biochemist, two researchers in the area of diabetes in pregnancy, one epidemiologist, two obstetricians and four endocrinologists. The participants represented ten countries and three continents. Before the discussion on each outcome, the participants were shown the previous voting results on that particular outcome by the total group and by each stakeholder group. Each outcome was discussed and there was agreement that some items should be grouped and/or rephrased. Therefore, " $75 \mathrm{~g}$ oral glucose tolerance test', 'Blood glucose level at $2 \mathrm{~h}$ during the $75 \mathrm{~g}$ oral glucose tolerance test', 'Fasting glucose' and ' $\mathrm{HbA}_{1 \mathrm{c}}$ blood levels' were combined into 'Assessment of glycaemic status'. 'Type 2 diabetes' became 'Diagnosis of type 2 diabetes since the index pregnancy'. 'GDM in subsequent/future pregnancies' became 'Number of pregnancies with a diagnosis of GDM since the index pregnancy'. 'Impaired fasting glucose' and 'Impaired glucose tolerance' were combined into 'Diagnosis of prediabetes since the index 
Table 1 Characteristics of participants in the Delphi online survey

\begin{tabular}{|c|c|c|c|}
\hline Variable & Round $1, \%(n=288)$ & Round 2, \% $(n=190)$ & Round 3, \% $(n=165)$ \\
\hline Sex (female) & 73 & 73.7 & 72.1 \\
\hline \multicolumn{4}{|l|}{ Experience/background } \\
\hline Diabetes nurse specialist & 5.5 & 6.3 & 5.5 \\
\hline Endocrinologist & 31.2 & 35.8 & 36.4 \\
\hline Obstetrician & 14.2 & 15.8 & 17.6 \\
\hline Paediatrician & 1.7 & 2.1 & 0.6 \\
\hline Neonatologist & 1.7 & 1.6 & 1.2 \\
\hline Midwife & 5.5 & 4.7 & 4.8 \\
\hline General practitioner & 3.8 & 3.1 & 3 \\
\hline Practice nurse & 0.3 & 0.5 & 0.6 \\
\hline Dietitian & 1.7 & 1 & 0.6 \\
\hline Physiotherapist & 1 & 0 & 0 \\
\hline Other & 11.8 & 11.6 & 12.1 \\
\hline Woman with previous diagnosis of GDM & 14.2 & 10 & 10.3 \\
\hline Researcher with expertise in diabetes & 5.5 & 5.8 & 6.1 \\
\hline Policy maker & 1.4 & 1.6 & 0.6 \\
\hline Clinician & 78.9 & 82.6 & 83.3 \\
\hline Health service user & 14.2 & 10 & 10.3 \\
\hline Researcher/policy maker & 6.9 & 7.4 & 6.7 \\
\hline \multicolumn{4}{|l|}{ Participant's country of residence } \\
\hline Albania & 0.3 & 0.5 & 0.6 \\
\hline Argentina & 7.3 & 6.8 & 6.7 \\
\hline Australia & 3.1 & 4.2 & 3.6 \\
\hline Austria & 0.7 & 1.1 & 1.2 \\
\hline Belgium & 1 & 0.5 & 0.6 \\
\hline Canada & 1 & 1.6 & 1.8 \\
\hline Chile & 0.3 & 0.5 & 0.6 \\
\hline Croatia & 0.7 & 1.1 & 1.2 \\
\hline Czech Republic & 0.3 & 0 & 0 \\
\hline Denmark & 3.8 & 4.2 & 4.8 \\
\hline France & 1.3 & 2.1 & 2.4 \\
\hline Germany & 1 & 0.5 & 0.6 \\
\hline Greece & 0.7 & 1.1 & 0.6 \\
\hline Hungary & 0.3 & 0 & 0 \\
\hline India & 0.7 & 0.5 & 0.6 \\
\hline Ireland & 49.8 & 47.4 & 49 \\
\hline Israel & 0.7 & 0 & 0 \\
\hline Italy & 2.1 & 2.6 & 2.4 \\
\hline Jamaica & 0.3 & 0.5 & 0.6 \\
\hline Lithuania & 1.4 & 1.6 & 1.8 \\
\hline Malta & 0.3 & 0.5 & 0.6 \\
\hline The Netherlands & 0.7 & 1.1 & 0.6 \\
\hline New Zealand & 3.8 & 4.2 & 3 \\
\hline Nigeria & 0.3 & 0.5 & 0.6 \\
\hline Poland & 0.7 & 0 & 0 \\
\hline Portugal & 0.3 & 0.5 & 0 \\
\hline Romania & 0.7 & 1.1 & 1.2 \\
\hline Spain & 2.1 & 1.1 & 1.2 \\
\hline Sweden & 1 & 1.1 & 0.6 \\
\hline UAE & 0.7 & 0.5 & 0.6 \\
\hline UK & 7.4 & 6.4 & 4.8 \\
\hline USA & 4.2 & 5.8 & 6.8 \\
\hline Uruguay & 0.3 & 0.5 & 0 \\
\hline
\end{tabular}

UAE, United Arab Emirates

pregnancy' and 'Breastfeeding after the index pregnancy' became 'Breastfeeding'.

Following discussion, the panel voted on each outcome to determine whether it should or should not be included in the final set. The final COS included nine outcomes and is presented in Table 3, together with the percentage of participants that voted 'consensus in'.

\section{Discussion}

This study used robust methods to develop the first COS relevant to the follow-up of women with previous gestational diabetes treated with insulin and/or glucose-lowering agents. A Delphi consensus panel with 20 representatives from ten countries agreed on nine outcomes to be included in the final COS. It is 
Table 2 Percentage of round 2 participants $(n=190)$ scoring each outcome as $1-3,4-6$ or $7-9$ on the 9-point Likert scale

\begin{tabular}{|c|c|c|c|}
\hline Variable & Score $1-3, \%$ & Score $4-6, \%$ & Score $7-9, \%$ \\
\hline \multicolumn{4}{|l|}{ Laboratory tests } \\
\hline 75 g OGTT & 5 & 11 & 84 \\
\hline Glucose level at $30 \mathrm{~min}$ during the $75 \mathrm{~g}$ OGTT & 53 & 28 & 19 \\
\hline Glucose level at $1 \mathrm{~h}$ during the $75 \mathrm{~g}$ OGTT & 24 & 23 & 53 \\
\hline Glucose level at $2 \mathrm{~h}$ during the $75 \mathrm{~g}$ OGTT & 7 & 14 & 79 \\
\hline Glucose level at $3 \mathrm{~h}$ during the $75 \mathrm{~g}$ OGTT & 58 & 28 & 14 \\
\hline Insulin level at $30 \mathrm{~min}$ during the OGTT & 53 & 31 & 16 \\
\hline Insulin level at $2 \mathrm{~h}$ during the OGTT & 39 & 37 & 24 \\
\hline Fasting glucose & 3 & 15 & 82 \\
\hline Random glucose & 45 & 36 & 19 \\
\hline $\mathrm{HbA}_{1 \mathrm{c}}$ level & 4 & 13 & 83 \\
\hline Fasting $\mathrm{HbA}_{1 \mathrm{c}}$ & 50 & 20 & 30 \\
\hline Insulin level & 38 & 34 & 28 \\
\hline Fasting insulin level & 28 & 36 & 36 \\
\hline Insulin to glucose ratio & 28 & 40 & 32 \\
\hline Insulinogenic index & 30 & 42 & 28 \\
\hline Corrected insulin response & 37 & 40 & 23 \\
\hline Insulin sensitivity & 25 & 41 & 33 \\
\hline HOMA & 29 & 42 & 29 \\
\hline HOMA-IR & 26 & 39 & 35 \\
\hline HOMA- $\beta$ & 28 & 40 & 32 \\
\hline Triacylglycerol level & 24 & 36 & 40 \\
\hline Fasting triacylglycerol level & 12 & 33 & 55 \\
\hline Cholesterol level & 21 & 37 & 42 \\
\hline Fasting cholesterol & 14 & 37 & 49 \\
\hline HDL & 20 & 36 & 44 \\
\hline Fasting HDL & 15 & 38 & 47 \\
\hline LDL & 20 & 33 & 47 \\
\hline Fasting LDL & 16 & 36 & 48 \\
\hline Apo-lipoprotein B & 35 & 46 & 19 \\
\hline Oxidised lipoproteins & 38 & 46 & 16 \\
\hline ACR & 11 & 37 & 52 \\
\hline Insulin growth factor & 45 & 39 & 16 \\
\hline IGF-binding protein 3 level & 50 & 37 & 13 \\
\hline Paraoxonase & 54 & 33 & 13 \\
\hline Leptin & 40 & 40 & 20 \\
\hline Adiponectin & 39 & 42 & 19 \\
\hline Total circulating adiponectin & 43 & 42 & 15 \\
\hline High sensitivity $\mathrm{C}$-reactive protein & 35 & 45 & 20 \\
\hline Fibrinogen & 47 & 39 & 14 \\
\hline Alanine aminotransferase & 23 & 46 & 31 \\
\hline Thyroid stimulating hormone & 16 & 40 & 44 \\
\hline Free thyroxine & 28 & 35 & 37 \\
\hline Total tissue plasminogen activator antigen & 49 & 39 & 12 \\
\hline Cytokines & 47 & 41 & 12 \\
\hline Genotype for Pro12Ala polymorphism & 57 & 33 & 10 \\
\hline Plasma uric acid & 41 & 38 & 21 \\
\hline$\gamma$-glutamyl aminotransferase & 30 & 43 & 27 \\
\hline Vitamin D & 26 & 42 & 32 \\
\hline \multicolumn{4}{|l|}{ Clinical conditions } \\
\hline Type 2 diabetes & 0 & 3 & 97 \\
\hline Insulin-treated type 2 diabetes & 3 & 8 & 89 \\
\hline GDM in subsequent pregnancies & 0 & 4 & 96 \\
\hline The metabolic syndrome & 2 & 16 & 82 \\
\hline Impaired fasting glucose & 2 & 14 & 84 \\
\hline Impaired glucose tolerance & 2 & 14 & 84 \\
\hline Polycystic ovary syndrome & 4 & 26 & 70 \\
\hline Cardiovascular disease & 2 & 17 & 81 \\
\hline \multicolumn{4}{|l|}{ Physiological variables } \\
\hline Weight & 1 & 4 & 95 \\
\hline Height & 12 & 12 & 76 \\
\hline BMI & 1 & 13 & 86 \\
\hline Waist circumference & 2 & 18 & 80 \\
\hline Hip circumference & 9 & 34 & 57 \\
\hline Waist/hip ratio & 7 & 28 & 65 \\
\hline Body fat & 8 & 37 & 55 \\
\hline Lean mass & 7 & 45 & 48 \\
\hline Post-pregnancy weight retention & 2 & 24 & 74 \\
\hline $\mathrm{BP}$ & 1 & 11 & 88 \\
\hline Resting BP & 2 & 15 & 83 \\
\hline Systolic BP & 2 & 12 & 86 \\
\hline Diastolic BP & 2 & 12 & 86 \\
\hline Resting pulse & 6 & 38 & 56 \\
\hline
\end{tabular}


Table 2 (continued)

\begin{tabular}{|c|c|c|c|}
\hline Variable & Score $1-3, \%$ & Score $4-6, \%$ & Score $7-9, \%$ \\
\hline \multicolumn{4}{|l|}{ Diet and exercise } \\
\hline Coffee intake & 22 & 56 & 22 \\
\hline Tea intake & 25 & 55 & 20 \\
\hline Alcohol intake & 5 & 32 & 63 \\
\hline Protein intake & 12 & 45 & 43 \\
\hline Carbohydrate intake & 8 & 27 & 65 \\
\hline Fruit intake & 10 & 28 & 62 \\
\hline Vegetable intake & 9 & 29 & 62 \\
\hline Dairy intake & 8 & 39 & 52 \\
\hline Fat intake & 10 & 32 & 58 \\
\hline Fibre intake & 9 & 38 & 53 \\
\hline Low glycaemic intake & 10 & 35 & 55 \\
\hline Unsaturated fat intake & 13 & 32 & 55 \\
\hline Saturated fat intake & 12 & 31 & 57 \\
\hline Healthy fat intake & 14 & 29 & 57 \\
\hline Low fat intake & 18 & 40 & 42 \\
\hline Low carbohydrate high-fat diet & 17 & 37 & 46 \\
\hline Nutrition composition & 13 & 34 & 53 \\
\hline Food frequency & 13 & 29 & 58 \\
\hline Portion size & 7 & 27 & 66 \\
\hline Total caloric intake & 7 & 26 & 67 \\
\hline Sweetened beverage intake & 9 & 23 & 68 \\
\hline Physical activity & 2 & 9 & 89 \\
\hline Walking/cycling to work & 7 & 23 & 70 \\
\hline Time spent sitting & 6 & 23 & 71 \\
\hline Time spent sleeping & 9 & 26 & 65 \\
\hline \multicolumn{4}{|l|}{ Psychological variables } \\
\hline Quality of life & 3 & 18 & 79 \\
\hline Eating behaviour & 6 & 24 & 70 \\
\hline Adverse emotional status & 4 & 28 & 68 \\
\hline Mental health status & 4 & 23 & 73 \\
\hline Anxiety & 4 & 30 & 66 \\
\hline Depression & 4 & 23 & 73 \\
\hline Activity to enhance mood & 8 & 28 & 64 \\
\hline Postnatal depression & 5 & 15 & 80 \\
\hline \multicolumn{4}{|l|}{ Other } \\
\hline Smoking status & 4 & 7 & 89 \\
\hline Quality adjusted life year & 6 & 30 & 64 \\
\hline Healthcare costs & 11 & 30 & 59 \\
\hline Lost productivity due to absence from work & 11 & 39 & 50 \\
\hline Sickness resulting in absence from work & 11 & 37 & 52 \\
\hline Satisfaction with research participation & 15 & 41 & 44 \\
\hline Number of pregnancies since the index pregnancy & 6 & 17 & 77 \\
\hline Health insurance & 25 & 38 & 37 \\
\hline Income & 20 & 40 & 40 \\
\hline Menopause status & 17 & 35 & 48 \\
\hline Miscarriage after the index pregnancy & 11 & 29 & 60 \\
\hline Breastfeeding in the index pregnancy & 5 & 16 & 79 \\
\hline Breastfeeding at 1 year post delivery & 10 & 28 & 62 \\
\hline Plans for future pregnancies & 10 & 24 & 66 \\
\hline Employment status & 21 & 35 & 44 \\
\hline Return to work after pregnancy & 18 & 41 & 41 \\
\hline Current medications & 5 & 21 & 74 \\
\hline Oral contraceptive use & 14 & 23 & 63 \\
\hline Contraception method & 16 & 27 & 57 \\
\hline Hormone replacement therapy & 16 & 30 & 44 \\
\hline Hysterectomy & 25 & 35 & 40 \\
\hline Oophorectomy & 26 & 37 & 47 \\
\hline Genital malignancies & 20 & 30 & 40 \\
\hline
\end{tabular}

advised that all studies in this area use this COS to facilitate comparison among studies and limit heterogeneity and reporting bias. The application of agreed methods in developing a COS and the participation of multiple stakeholder groups assure the wide applicability and dissemination of this COS.

The wide applicability of the study was one of the main reasons why the outcomes ' $75 \mathrm{~g}$ oral glucose tolerance test', 'Blood glucose level at $2 \mathrm{~h}$ during the $75 \mathrm{~g}$ oral glucose tolerance test', 'Fasting glucose' and 'HbA $1 \mathrm{c}$ blood levels' were combined into 'Assessment of glycaemic status'; in so doing, the COS permits researchers the opportunity to assess glycaemic status according to their own national guidelines and resources. In addition, this COS identifies 'what is to be collected' and not 'how it is to be collected', which will be the subject of future work. Similarly, 'Impaired fasting glucose' and 'Impaired glucose tolerance' were combined as 
Table 3 Final outcomes to be included in the COS

\begin{tabular}{lc}
\hline Outcome & $\begin{array}{l}\text { Votes for the outcome } \\
\text { to be included in the } \\
\text { final COS, \% }\end{array}$ \\
\hline $\begin{array}{l}\text { Assessment of glycaemic status } \\
\text { Diagnosis of T2DM since the index pregnancy }\end{array}$ & 100 \\
Number of pregnancies since the & 90 \\
$\quad$ index pregnancy & 100 \\
Number of pregnancies with a diagnosis of GDM & 100 \\
$\quad$ since the index pregnancy & 100 \\
Diagnosis of prediabetes since the index & \\
pregnancy & 100 \\
BMI & 95 \\
Post-pregnancy weight retention & 100 \\
Resting BP & 75 \\
Breastfeeding &
\end{tabular}

T2DM, type 2 diabetes mellitus

'Diagnosis of prediabetes since the index pregnancy' to give the COS a worldwide applicability in light of the variability in diagnostic tools and criteria. However, while we would recommend that collection and reporting of all outcomes in the COS is mandatory, researchers can choose to collect any additional outcomes required for their study, including specific indices of glycaemic control.

Of importance, the COS consensus meeting had representation from a variety of health professionals/specialties, both local and international, and included women with a previous diagnosis of GDM. This stakeholder composition permitted both the health professionals and women to bring their experience and perspectives to the issues under discussion. Each participant was able to have an understanding of what was important to the other person and what was feasible. Ultimately, this resulted in shared decision-making in a study that will impact future research of women with gestational diabetes.

There are some limitations to our study. Currently, there are no methods for sample size calculation for this type of study. To minimise the potential for selection bias, participants were invited through international organisations and personal professional email lists. Only participants who completed each survey round were recorded. From the totality of initial emails sent before round 1 , we had a $35 \%$ response. This response rate, however, included participants from 33 countries and five continents. We had a $34 \%$ drop-off rate between round 1 and round 2, but, impressively, $87 \%$ of participants who completed round 2 also completed round 3 . There was a low response rate among primary care physicians $(3.5 \%)$.

Another potential limitation of this study is the large number of items to be scored in rounds 1 and 2 of the online Delphi survey, which may have impacted negatively on the survey response rate.

We had a large percentage of international participants, including individuals from high-income, upper- and lower-middleincome countries. However, low-income countries were underrepresented, and this may limit the generalisability of this COS to certain parts of the world. The participants at the consensus meeting tried to overcome this by rephrasing or combining outcomes in order to increase the worldwide applicability of the COS.

The access to service users was limited by data protection laws, so participants were recruited through the clinical facilities. Despite this, 30\% of respondents in round 1 were international service users. At the consensus meeting, 50\% of service user participants were non-Irish. We sought international participation for the COS to have global relevance. The service user representatives made important suggestions on all the outcomes discussed at the consensus meeting. Anonymous voting (via the electronic app during this meeting) aimed to prevent participants feeling compelled to vote in a certain way. It has been advocated that the views of health service users should be given greater value in the development of a COS [20] as outcomes reported for clinical studies might not reflect endpoints that are meaningful for them. Examples exist where health service users identified an outcome important to them as a group that might not have been considered by clinicians [21-23]. However, the responses from health service users and the responses from other stakeholder groups were generally concordant.

The strengths of the study include our use of robust methods in the COS development, including adherence to the COS-STAR statement, the thoroughness of the systematic review (six databases were searched for relevant studies), the high number of participants and the diversity of stakeholder groups participating at each stage of the COS.

Recent review papers have shown the degree of outcome reporting bias among studies, with the main outcome not being reported in up to $47 \%$ of the studies and inadequately reported in up to $76 \%$ of the studies reviewed [24-26]. Therefore, there is a cogent argument for creating and disseminating a COS to harmonise outcome reporting.

This is the first study to outline a COS for the long-term follow-up of women with previous GDM. We encourage all investigators undertaking research in this field to report, as a minimum, this COS to reduce reporting bias by allowing evidence synthesis across clinical studies. This will ultimately lead to improvements in the quality of research and delivery of evidence-based healthcare for women with GDM.

Acknowledgements We thank all the stakeholders who participated in the Delphi study and consensus meeting. We thank Wellcome and Health Research Board for funding the Irish Clinical Academic Training (ICAT) Programme, of which DB is a training Fellow.

Data availability Data are available on request.

Funding The HRB-Trials Methodology Research Network part funded the consensus meeting. TPG is supported by a Hardiman Scholarship from the College of Medicine, Nursing and Health Science, National University of Ireland, Galway, Ireland, and a bursary from the Irish Endocrine Society/Royal College of Physicians of Ireland.

Duality of interest The authors declare that there is no duality of interest associated with this manuscript. 
Contribution statement DB drafted the manuscript, analysed the data and coordinated revisions. All authors participated in and made substantial contribution to the study design or acquisition of data and revised the manuscript critically for important intellectual content. All authors read and approved the final manuscript. DB is responsible for the integrity of the work as a whole.

Open Access This article is distributed under the terms of the Creative Commons Attribution 4.0 International License (http:// creativecommons.org/licenses/by/4.0/), which permits unrestricted use, distribution, and reproduction in any medium, provided you give appropriate credit to the original author(s) and the source, provide a link to the Creative Commons license, and indicate if changes were made.

\section{References}

1. Guariguata L, Linnenkamp U, Beagley J, Whiting DR, Cho NH (2014) Global estimates of the prevalence of hyperglycaemia in pregnancy. Diabetes Res Clin Pract 103(2):176-185. https://oi. org/10.1016/j.diabres.2013.11.003

2. O'Sullivan EP, Avalos G, O'Reilly M et al (2011) Atlantic Diabetes in Pregnancy (DIP): the prevalence and outcomes of gestational diabetes mellitus using new diagnostic criteria. Diabetologia 54(7):1670-1675. https://doi.org/10.1007/s00125-011-2150-4

3. Melchior H, Kurch-Bek D, Mund M (2017) The prevalence of gestational diabetes. Dtsch Arztebl Int 114:412-418. https://doi. org/10.3238/arztebl.2017.0412

4. Farrar D, Simmonds M, Bryant M et al (2017) Treatments for gestational diabetes: a systematic review and meta-analysis. BMJ Open 7(6):e015557. https://doi.org/10.1136/bmjopen-2016015557

5. Bellamy L, Casas JP, Hingorani AD, Williams D (2009) Type 2 diabetes mellitus after gestational diabetes: a systematic review and metaanalysis. Lancet 373(9677):1773-1779. https://doi.org/10. 1016/S0140-6736(09)60731-5

6. Hakkarainen H, Huopio H, Cederberg H, Pääkkönen M, Voutilainen R, Heinonen S (2016) The risk of metabolic syndrome in women with previous GDM in a long-term follow-up. Gynecol Endocrinol 32(11):920-925. https://doi.org/10.1080/09513590. 2016.1198764

7. Kramer CK, Campbell S, Retnakaran R (2019) Gestational diabetes and the risk of cardiovascular disease in women: a systematic review and meta-analysis. Diabetologia 62(6):905-914. https://doi. org/10.1007/s00125-019-4840-2

8. Kozhimannil KB, Pereira MA, Harlow BL (2009) Association between diabetes and perinatal depression among low-income mothers. JAMA 301(8):842-847. https://doi.org/10.1001/jama. 2009.201

9. Dalfrà $\mathrm{MG}$, Nicolucci $\mathrm{A}$, Bisson $\mathrm{T}$, Bonsembiante $\mathrm{B}$, Lapolla $\mathrm{A}$, QLISG (Quality of Life Italian Study Group) (2012) Quality of life in pregnancy and post-partum: a study in diabetic patients. Qual Life Res 21(2):291-298. https://doi.org/10.1007/s11136-0119940-5

10. Wartko PD, Beck TL, Reed SD, Mueller BA, Hawes SE (2017) Association of endometrial hyperplasia and cancer with a history of gestational diabetes. Cancer Causes Control 28(8):819-828. https:// doi.org/10.1007/s10552-017-0908-9

11. American Diabetes Association (2015) 12. Management of diabetes in pregnancy. Diabetes Care 38(Suppl 1):S77-S79. https://doi.org/ $10.2337 / \mathrm{dc} 15-\mathrm{S} 015$
12. Gargon E, Williamson PR, Altman DG, Blazeby JM, Tunis S, Clarke M (2017) The COMET Initiative database: progress and activities update. Trials 18(1):54. https://doi.org/10.1186/s13063017-1788-8

13. Gargon E, Williamson PR, Altman DG, Blazeby JM, Clarke M (2014) The COMET initiative database: progress and activities from 2011 to 2013. Trials 15(1):279. https://doi.org/10.1186/ 1745-6215-15-279

14. Kirkham JJ, Gorst S, Altman DG et al (2016) Core Outcome SetSTAndards for reporting: the COS-STAR statement. PLoS Med 13(10):e1002148. https://doi.org/10.1371/journal.pmed.1002148

15. Ziegler AG, Wallner M, Kaiser I et al (2012) Long-term protective effect of lactation on the development of type 2 diabetes in women with recent gestational diabetes mellitus. Diabetes 61(12):31673171. https://doi.org/10.2337/db12-0393

16. Xu Y, Shen S, Sun L, Yang H, Jin B, Cao X (2014) Metabolic syndrome risk after gestational diabetes: a systematic review and meta-analysis. PLoS One 9(1):e87863. https://doi.org/10.1371/ journal.pone. 0087863

17. Core Outcome Measures in Effectiveness Trials (COMET) Initiative database. Available from www.comet-initiative.org. Accessed 9 May 2018

18. Dalkey NC (1969) The Delphi Method: an experimental study of group opinion. Rand Corp Public RM-58888-PR. Rand Corp, Santa Monica

19. Bogdanet D, Egan A, Fhelelboom N et al (2019) Metabolic followup at one year and beyond of women with gestational diabetes treated with insulin and/or oral hypoglycaemic agents: study protocol for the identification of a core outcomes set using a Delphi survey. Trials 20(1):9. https://doi.org/10.1186/s13063-018-3059-8

20. Williamson PR, Altman DG, Blazeby JM et al (2012) Developing core outcome sets for clinical trials: issues to consider. Trials 13(1): 132. https://doi.org/10.1186/1745-6215-13-132

21. Sinha I, Gallagher R, Williamson PR, Smyth RL (2012) Development of a core outcome set for clinical trials in childhood asthma-a survey of clinicians, parents and young people. Trials 13(1). https://doi.org/10.1186/1745-6215-13-103

22. Kirwan JR, Hewlett SE, Heiberg T et al (2005) Incorporating the patient perspective into outcome assessment in rheumatoid arthritisprogress at OMERACT 7. J Rheumatol 32:2250-2256

23. Oliver S, Gray J (2006) A bibliography of research reports about patients', clinicians' and researchers' priorities for new research. James Lind Alliance, London

24. Saini P, Loke YK, Gamble C, Altman DG, Williamson PR, Kirkham JJ (2014) Selective reporting bias of harm outcomes within studies: findings from a cohort of systematic reviews. BMJ 349: g6501. https://doi.org/10.1136/bmj.g6501

25. Page MJ, McKenzie JE, Kirkham J et al (2014) Bias due to selective inclusion and reporting of outcomes and analyses in systematic reviews of randomised trials of healthcare interventions. Cochrane Database Syst Rev MR000035. https://doi.org/10.1002/14651858. MR000035.pub2

26. Smith V, Clarke M, Williamson P, Gargon E (2015) Survey of new 2007 and 2011 Cochrane reviews found 37\% of prespecified outcomes not reported. J Clin Epidemiol 68(3):237-245. https://doi. org/10.1016/j.jclinepi.2014.09.022

Publisher's note Springer Nature remains neutral with regard to jurisdictional claims in published maps and institutional affiliations. 


\section{Affiliations}

Delia Bogdanet ${ }^{1}$ (D) Catriona Reddin ${ }^{1} \cdot$ Esther Macken $^{1} \cdot$ Tomas P. Griffin $^{1} \cdot$ Narjes Fhelelboom $^{1} \cdot$ Linda Biesty $^{1}$. Shakila Thangaratinam ${ }^{2} \cdot$ Eugene Dempsey $^{3} \cdot$ Caroline Crowther $^{4} \cdot$ Sander Galjaard $^{5} \cdot$ Michael Maresh $^{6}$. Mary R. Loeken ${ }^{7,8}$. Angela Napoli ${ }^{9}$ - Eleni Anastasiou ${ }^{10}$ - Eoin Noctor ${ }^{11} \cdot$ Harold W. de Valk $^{12}$. Mireille N. M. van Poppel ${ }^{13} \cdot$ Andrea Agostini $^{14}$. Cheril Clarson ${ }^{15,16} \cdot$ Aoife M. Egan ${ }^{17}$ • Paula M. O'Shea ${ }^{1}$. Declan Devane ${ }^{1} \cdot$ Fidelma P. Dunne ${ }^{1}$

1 College of Medicine, Nursing and Health Sciences, National University Ireland, University Road, Galway H91 TK33, Ireland

2 Queen Mary, University of London, Women's Health Research Unit, London, UK

3 INFANT Centre and Department of Paediatrics \& Child Health, University College Cork, Cork, Ireland

4 Liggins Institute, The University of Auckland, Auckland, New Zealand

5 Department of Obstetrics and Gynaecology, Division of Obstetrics and Prenatal Medicine, Erasmus MC, University Medical Centre Rotterdam, Gravendijkwal 230, 3015 CE Rotterdam, the Netherlands

6 Department of Obstetrics, St Mary's Hospital, Manchester University Hospitals NHS Foundation Trust, Manchester Academic Health Science Centre, Manchester, UK

7 Section of Islet Cell and Regenerative Biology, Joslin Diabetes Center, Boston, MA, USA
8 Department of Medicine, Harvard Medical School, Boston, MA, USA

9 Department of Clinical and Molecular Medicine, Sant'Andrea University Hospital, Sapienza, University of Rome, Rome, Italy

10 Department of Endocrinology, Metabolism and Diabetes Centre, Alexandra Hospital, Athens, Greece

11 Department of Endocrinology, University Hospital Limerick, Limerick, Ireland

12 Department of Internal Medicine, University Medical Centre Utrecht, Utrecht, the Netherlands

13 Institute of Sport Science, University of Graz, Graz, Austria

14 A.S.L Viterbo Distretto A, Consultorio Montefiascone, Rome, Italy

15 Department of Pediatrics, University of Western Ontario, London, ON, Canada

16 Lawson Health Research Institute, London, ON, Canada

17 Division of Endocrinology, Diabetes and Metabolism, Mayo Clinic, Rochester, MN, USA 